Adult neurogenesis has been suggested to support the recall of temporally related memories ${ }^{2}$ : the enhanced excitability of newly generated young granule cells could result in a larger rate of activation during exploration and memory encoding. The larger activity within the population of young neurons might produce substantial overlap of activity patterns representing different but temporally related events. Overlapping patterns might interfere with each other during recall, leading to 'false positive' temporally related memories ${ }^{2}$. The new data from Kee et al. ${ }^{3}$ indeed show that the proportion of active newborn cells is two to three times larger than that of old granule cells. However, a total fraction of about $5 \%$ of active cells is still a sparse distributed representation. Furthermore, young neurons seem to support, rather than compromise, the separation of memories for similar objects in a delayed, nonmatched-to-sample task $^{13}$ or in an object-recognition task ${ }^{14}$.

So what are adult-born neurons good for? The nonoverlapping orthogonal representation in a competitive network is achieved by categorization of input patterns ${ }^{10}$. Because associative synaptic plasticity will be induced due to input correlations, the strength of synaptic connections is adjusted in such a way that 'characteristic' features activate a small subpopulation of neurons in a winner-takesall fashion. However, this might be difficult with the powerful dendritic inhibition shown for dentate granule cells in vivo during exploratory activity ${ }^{15}$. The enhanced plasticity of newly generated young granule cells might facilitate feature learning during exploration. As old granule cells show a higher threshold for synaptic plasticity, the connectivity might become relatively stable later on ${ }^{8}$. As a consequence, mature neurons would be most sensitive to features they learned when they were young. Adding new neurons could help the network to achieve both stable analysis of 'old' features as well as adaptation to new environments, finally supporting precise and distinct representations of new memories throughout life.

To decide between these partially opposing views, future experiments are required. What happens with a reduced number of training sessions? It would be very interesting to see whether a failure in memory retention is correlated with a reduced or enhanced activity in the new neurons. Are new cells more active when animals have to distinguish similar input patterns? This could be studied using cellular compartment analysis of temporal activity by fluorescence in situ hybridization (catFISH)9. How much overlap between activity patterns will occur in environments that are similar or in different environments that were explored in the past at approximately the same time point? Although there are still many open questions, the results of Kee et al. represent a significant advance in the understanding of the functional role of adult neurogenesis in the mammalian central nervous system.

\section{COMPETING INTERESTS STATEMENT}

The author declares that he has no competing financial interests.

1. Squire, L.R., Stark, C.E. \& Clark, R.E. Annu. Rev. Neurosci. 27, 279-306 (2004).

2. Aimone, J.B., Wiles, J. \& Gage, F.H. Nat. Neurosci. 9 723-727 (2006).

3. Kee, N., Teixeira, C.M., Wang, A.H. \& Frankland, P.W. Nat. Neurosci. 10, 355-362 (2007).

4. Song, H. et al. J. Neurosci. 25, 10366-10368 (2005).

5. Espósito, M.S. et al. J. Neurosci. 25, 10074-10086 (2005).

6. Ge, S. et al. Nature 439, 589-593 (2006).

7. Zhao, C., Teng, E.M., Summers, R.G., Ming, G. \& Gage, F.H. J. Neurosci. 26, 3-11 (2006).

8. Schmidt-Hieber, C., Jonas, P. \& Bischofberger, J. Nature 429, 184-187 (2004)

9. Chawla, M.K. et al. Hippocampus 15, 579-586 (2005).

10. Rolls, E. \& Treves, A. Neural Networks and Brain Function (Oxford University Press, Oxford, 1998).

11. Ramirez-Amaya, V., Marrone, D.F., Gage, F.H., Worley, P.F. \& Barnes, C.A. J. Neurosci. 26, 12237-12241 (2006).

12. Amaral, D.G., Ishizuka, N. \& Claiborne, B. Prog. Brain Res. 83, 1-11 (1990).

13. Winocur, G., Wojtowicz, J.M., Sekeres, M., Snyder, J.S. \& Wang, S. Hippocampus 16, 296-304 (2006).

14. Bruel-Jungerman, E., Laroche, S. \& Rampon, C. Eur. J. Neurosci. 21, 513-521 (2005).

15. Moser, E.I. J. Neurosci. 16, 1247-1259 (1996).

\title{
Unraveling the mechanisms of Angelman Syndrome
}

Angelman Syndrome (AS) is a genetic disorder that is characterized by movement disorders, seizures and mental retardation. Although relatively rare (affecting 1 in 15,000 people), this disorder is currently untreatable. On page 280 , van Woerden and colleagues report that aberrant phosphorylation of calcium/calmodulin-dependent kinase type 2 (CaMKII) is the main molecular dysfunction underlying the neurological phenotype of AS.

AS is caused by the loss of normal maternal contribution to a region of chromosome 15 . This region contains a gene, $U B E 3 A$, encoding a ubiquitin protein ligase. Although the function of this protein is not known, the gene has different patterns of methylation in the paternal and maternal chromosomes. One of these gene copies is silenced; in the hippocampus and cerebellum, the maternal allele is the one that is usually active. A deletion in the maternal allele of $U B E 3 A$ therefore leads to a loss of $U B E 3 A$ expression in these regions. Mice with a maternally inherited $U B E 3 A$ mutation show seizures and cognitive abnormalities similar to those seen in humans with AS.

These AS mice also show decreased activity of CaMKII, known to be important for neuronal function. Van Woerden and colleagues now report that if they cross AS mice with an CaMKII mutant that is unable to undergo phosphorylation at the Thr305/306 site (which prevents selfinhibition of CaMKII), they can rescue all the main features of AS in these mice. The AS/CaMKII double mutants show a reduction of seizure propensity compared to AS mice and also show normal motor behavior. Whereas the AS mice have increased body weight, the double mutants have normal weight. Moreover, the deficits in long-term potentiation seen in the AS mice are ameliorated in the AS/CaMKII mutants. Cognitive deficits associated with AS mice, such as defects in spatial learning and fear conditioning, are also rescued by introducing this CaMKII mutation. These findings show conclusively that increased inhibitory phosphorylation of CaMKII is responsible for many of the neurological deficits observed in AS.

Kalyani Narasimhan
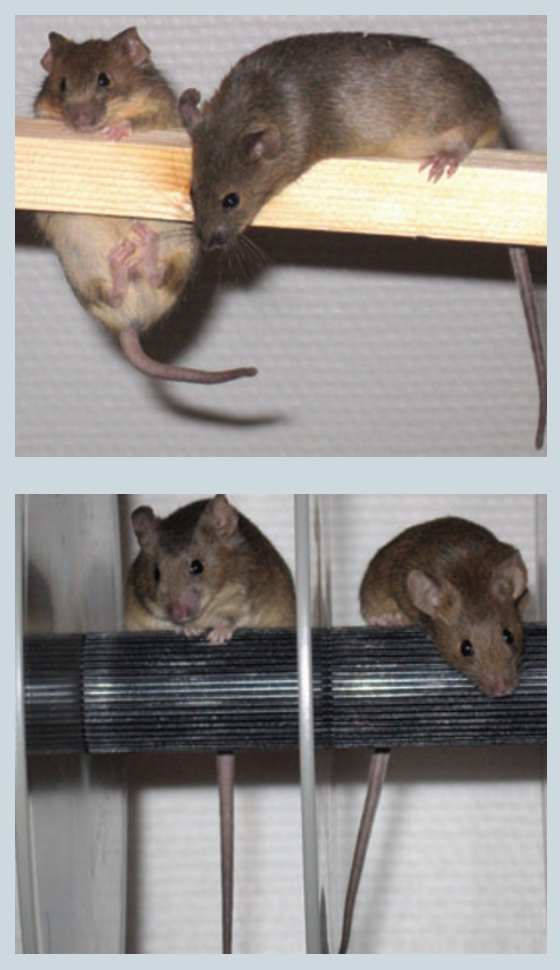